remains unproved. The series of Paterson et al contains too little clinical information - 39 patients over 10 years were diagnosed, seven infants sustained fractures in hospital (were these the $21 \%$ born at less than 33 weeks gestation?). ${ }^{6}$ The fractures were numerous, rib fractures were common as were metaphyseal abnormalities and periosteal reactions. Three had fractures at birth, and most at less than 4 months. How the child came to have radiographs taken is not clear or how physical abuse was excluded. The picture has similarities to the copper or temporary copper deficiency story which was put into perspective by Shaw in $1988 .{ }^{7}$

Dr Smith concludes by acknowledging that osteogenesis imperfecta is rare. He might have added that osteogenesis imperfecta with no family history of fractures, joint laxity, early onset deafness, blue sclerae, and dentinogenesis is very rare. In addition the probability that an individual infant with no relevant family history has osteogenesis imperfecta where the skeleton is normal, there are no wormian bones, there is no or trivial history of trauma, the infant is not weight bearing yet has a fractured skull, ribs, or metaphyseal fractures is in the Taitz range of probabilities, that is, millions.

Collagen analysis is clearly important in understanding the pathogenesis of osteogenesis imperfecta, but until the correlation with clinical disorder is more precise it is difficult to see the biopsy report being more than another piece of the jigsaw, that is, not a diagnostic test but offering some corroboration of non-abuse. The diagnosis of possible osteogenesis imperfecta can not wait three months but a multicentre study looking at fractures in infancy (paediatrically, radiologically, orthopaedically, and biochemically) would be the way forward to sort out the real from the supposed disorder.

JANE $M$ WYNNE CHRISTOPHER J HOBBS Leeds Community and Mental Health Unit Belmont House,

3/5 Belmont Grove Leeds LS2 9NP

1 Ablin DS, Greenspan A, Reinhart M, Grix A. Differentiation of child abuse from osteogenesis imperfecta. $A f R \quad 1990$; 154: $1035-46$.

2 Taitz LS. Child abuse and osteogenesis imperfecta. $B M \mathcal{F}$ 1987; 295: 1082-3.

3 Hobbs CJ. Physical abuse. In: Hobbs CJ, Hanks HGI, Wynne JM, eds. Child abuse and neglect. Edinburgh Churchill Livingstone, 1993: 47-75.

4 Carty H. Brittle or battered. Arch Dis Child 1988; 63: 350-2.

Astley R. Metaphyseal fractures in osteogenesis imperfecta. Br $\mathcal{F}$ Radiol 1979; 52: 441-3.

6 Paterson CR, Burns J, McAllion SJ. Osteogenesis imperfecta: the distinction from child abuse and the recognition of variant form. Am $\mathcal{F}$ Med Genet 1993; 45: 187-92.

7 Shaw JCL. Copper deficiency and non-accidental injury. Arch Dis Child 1988; 63: 446-55.

\section{Commentary (2)}

This review has been invited to try and establish the parameters for making the diagnosis of osteogenesis imperfecta in order to avoid an erroneous diagnosis of NAI and to review the evidence for the most recently suggested hypothesis, temporary brittle bone disease, ${ }^{1}$ put forward as an explanation in contested cases of NAI. Has the article achieved its objectives and how does it take the debate forward? I offer the following comments.

The level of general knowledge about current concepts in osteogenesis imperfecta is very varied within paediatrics, and developments in molecular genetics in establishing the presence of abnormal collagen in up to $85 \%$ of patients with osteogenesis imperfecta are perhaps not as widely appreciated as they should be. The Sillence classification remains the one most understood within paediatrics. Dr Smith indicates that not all patients fit comfortably within the mould. We still await a definitive article in the general paediatric literature, explaining new thoughts on osteogenesis imperfecta classification, broadly referenced from several sources. We also need to understand why $15-20 \%$ of patients with osteogenesis imperfecta do not have the gene for abnormal collagen. Is there another genetic problem as yet undiagnosed? Smith states that the normal population do not have abnormal collagen but the statement is unreferenced. A reference indicating the basis for this would be helpful. A greater general understanding of the genetics of osteogenesis imperfecta, and the place of other collagen disorders demonstrable by fibroblast culture in relation to osteogenesis imperfecta, is of immense importance. There are still many unanswered questions.

Dr Smith indicates that recent reviews indicate that type IV osteogenesis imperfecta, and I presume he refers to both types $\mathrm{A}$ and $\mathrm{B}$, is more frequent than thought. That statement is unreferenced. We need to know the evidence. It would be extremely helpful to know the incidence and natural history of type IV A osteogenesis imperfecta, the form usually suggested in contested cases of abuse, quoting references other than Paterson and Taitz. ${ }^{2-4}$ In Sillence's paper ${ }^{4}$ he indicated then that the radiology of the skeleton in type IV has not been well defined, the bone changes were severe, and he comments that there is no known feature differentiating type IV from osteogenesis imperfecta type III. Since then, it has been suggested that the radiographic features are more variable. ${ }^{1}$

Paterson's diagnosis of type IV A osteogenesis imperfecta has been questioned on the basis of his methodology.5-7 Therefore, we need independent sources. Taitz's calculation of the incidence of new mutations is the standard reference. ${ }^{3}$ Is this accepted as accurate by geneticists, for, if so, then the possibility of a wrong diagnosis being made in contested cases becomes so unlikely that it hardly merits discussion?

Dr Smith indicates that no type of fracture excludes osteogenesis imperfecta and that infants with it may sustain any type of fracture in a skeleton that is radiologically otherwise normal. What is the evidence for this? Children with severe changes of osteogenesis imperfecta may have multiple fractures affecting almost anywhere, excepting the skull, unless there is coincidental trauma. Children with mild disease, on the contrary, mainly sustain 
fractures of the long bones in response to trauma that would not normally cause a fracture in a child with normal bones, and are brought to medical attention because of the injury, which is usually solitary, and it is rare to find further injury on skeletal survey. This is my personal clinical experience and corroborated by colleagues. The age of clinical presentation of osteogenesis imperfecta with the first fracture is variable, but tends to be when the infant becomes mobile or in early childhood. If the injury is unobserved, then the child may present with an unexplained fracture and it is in these circumstances that the question of NAI is inevitably raised, especially when the fracture is solitary. Multiple fractures and rib fractures are not features of mild osteogenesis imperfecta. It must also be remembered that the fractures of osteogenesis imperfecta are painful, hence the clinical presentation. It is sometimes stated that as the NAI fractures detected on skeletal survey are occult and have not been observed clinically, that this is evidence for osteogenesis imperfecta, as it is suggested that the easy fracturing of osteogenesis imperfecta is not painful. When clinical signs of osteogenesis imperfecta are present, or there is an appropriate family history, a wrong diagnosis should not occur. In the absence of clinical features and a family history, the explanation usually suggested is a mutation. Smith quotes the incidence of osteogenesis imperfecta as one in 20000 . This presumably means all types, including those with a family history. Hard information on spontaneous mutation rates for the different types of osteogenesis imperfecta is not quoted.

Dr Smith indicates that controversy exists about the significance of metaphyseal fractures but does not reference his statement to this or his comment about skull fractures in osteogenesis imperfecta. One assumes he is referring to Paterson's publications. Astley reported metaphyseal fractures in osteogenesis imperfecta but concluded that the bone disease of osteogenesis imperfecta was so obvious that one could not mistake these for NAI. ${ }^{8}$ One can also get metaphyseal spurs and chip fractures that could simulate NAI in rickets, scurvy, copper deficiency, and Menkes' syndrome, but in these cases there is clinical, chemical and radiological evidence of disease and a wrong diagnosis should not occur. Corner metaphyseal fractures of the type found in NAI do not occur in mild osteogenesis imperfecta. Fractures within the metaphysis close to its junction with the diaphysis can occur in osteogenesis imperfecta or in the normal toddler. The case illustrated by Paterson et al (fig 1 in their paper) is typical of one of these fractures and is not the corner fracture of NAI. ${ }^{1}$ This has been pointed out in a letter to the editor. ${ }^{9}$

Skull fractures in children with osteogenesis imperfecta occur as the result of trauma. Intracranial trauma is rare. There are only two references identified during a literature search as to its occurrence. ${ }^{1011}$ Therefore, the finding of incracranial injury in a child with bone injury must raise the question of NAI.
TEMPORARY BRTTTLE BONE DISEASE

Dr Smith states that the existence of this condition is not in doubt but quotes no reference to this statement. I assume he is basing this on the publication by Paterson et al. ${ }^{1}$ This paper warrants careful reading. In it the authors refer to a self limiting form of osteogenesis imperfecta, which they term temporary brittle bone disease, thus confusing osteogenesis imperfecta with its genetic implications, with a stated hypothesis that they 'think that this disorder reflects a temporary collagen defect, probably caused by a temporary deficiency of an enzyme, possibly a metalloenzyme involved in a post-translational processing of collagen': three postulates in one sentence. They describe 39 patients over a 10 year period with fractures and clinical features indistinguishable from NAI and quote comparison between these children and their own cohort of patients in whom they reported copper deficiency. ${ }^{12}$ There is no reference in this paper to that of Shaw who indicated in this full review that the incidence of copper deficient bone disease is very rare. ${ }^{13}$ This contrasts with Paterson's experience. Similar remarks by Ablin et $a l^{5}$ and Carty and Shaw ${ }^{14}$ apply to his large cohort of patients with osteogenesis imperfecta type IV. ${ }^{2}$ The contrasting experience of Paterson, with his cohort of patients as compared to that in the rest of the published literature, should suggest caution until further similar series are published.

Does a temporary form of brittle bone disease exist? Currently it is purely a hypothesis and one that is put forward in contested cases of NAI. The explanation offered as to why the children do not sustain further injury when removed from the place of injury is that they have grown out of the disease (personal communication).

How does all this fit in with our knowledge of the skeletal and cerebral injuries of NAI, garnered now over a period of almost 50 years since Caffey first put forward his postulate. The evidence for NAI as the cause of the multiplicity of injuries is strong, supported by the evidence of assailants, ${ }^{15}$ painstaking histopathological studies in infants dying as the result of abuse, ${ }^{16}$ numerous comparative analyses of the injuries of abuse versus accidental trauma, and numerous admissions of abuse (personal experience). These children must also be seen in balance with the everyday pattern of illness as seen by paediatricians and paediatric radiologists so that a balanced perspective is maintained when faced with a case. That one wants to find an alternative explanation for the injuries sustained by these children is understandable but a court of law is not the place to debate medical hypotheses. ${ }^{17}$

Dr Smith offers two ways forward in children in whom a diagnosis of NAI is contested, fibroblast culture and a wait and see policy. Fibroblast culture takes at least three months to produce an answer, so offers no speedy solution. Should it be done on all query NAI children or only those in whom the issue is contested? What are the implications for the blanket policy in terms of cost, laboratory 
facilities, and personnel? Why should some children have it and not others? Who is going to draw up guidelines?

I agree that if, during the wait and see period, the child develops further injury while in a place of safety, or overt features of osteogenesis imperfecta, then the diagnosis of osteogenesis imperfecta, real and not temporary, must be strongly considered. If, during this period, a diagnosis of osteogenesis imperfecta becomes clear, a great injustice has been done to that family. This is the dilemma for paediatricians and radiologists. If, however, no further fractures occur, then this is traditionally regarded as further evidence that the initial injury was NAI. What is the likelihood of this happening in true osteogenesis imperfecta or the disputed temporary brittle bone disease?

The article has highlighted many issues but leaves us with many unanswered questions.

HELEN CARTY Department of Radiology, Royal Liverpool Children's Hospital NHS Trust, Alder Hey, Eaton Road, Liverpool L12 2AP

1 Paterson CR, Burns J, McAllion SJ. Osteogenesis imperfecta: the distinction from child abuse and the imperfecta: the distinction from child abuse and the 187-92.

2 Paterson CR, McAllion SJ, Shaw JW. Clinical and radiological features of osteogenesis imperfecta type IV A. Acta Paediatr Scand 1987; 76: 548-52.

3 Taitz L. Child abuse and osteogenesis imperfecta. $B M \mathcal{F}$ 1987; 295: 1082-3.

4 Sillence D. Osteogenesis imperfecta: an expanding panorama of variants. Clin Orthop 1981; 159: 11-25.

5 Ablin DS, Greenspan A, Reinhart M, Grix A. Differentiation of child abuse from osteogenesis imperfecta. $A f R$ 1990; 154: 1035-46.

6 Carty $H$. Differentiation of child abuse from osteogenesis imperfecta [Letter]. $A \mathcal{F}$ 1991; 156: 635.

7 Carty H, Shaw D. Child abuse and osteogenesis imperfecta [Letter]. BMF 1988; 296: 292.

8 Astley R. Metaphyseal fractures in osteogenesis imperfecta. Br $₹$ Radiol 1979; 52: 441-3.

9 Shaw D, Hall C, Carty H. Osteogenesis imperfecta: the distinction from child abuse and the recognition of the distinction from child abuse and the reco
variant form. $A m \mathcal{F}$ Med Genet (in press).

10 Pozzati E, Popp M, Gaist G. Acute bilateral extradural hematomas in a case of osteogenesis imperfecta congenita. Neurosurgery 1983; 13: 66-8.

11 Tokoro K, Nakajima F, Yamataki A. Infantile chronic subdural hematoma with local protrusion of the skull in a case of osteogenesis imperfecta. Neurosurgery 1988; 22: 595-8.

12 Paterson CR, Burns J. Copper deficiency in infancy. Fournal of Clinical Biochemical Nutrition 1988; 4: 175-90.

13 Shaw JCL. Copper deficiency and non-accidental injury. Arch Dis Child 1988; 63: 448-55.

14 Carty H, Shaw DG. Osteogenesis imperfecta type IV A Acta Paediatr Scand 1988; 77: 752-4.

15 Kleinman P. Diagnostic imaging in child abuse. AfR 1990;

155: 703-12.
16 Kleinman $P$. The metaphyseal lesion in abused infants: a radiologic-histopathologic study. $A \mathcal{F R}$ 1986; 146: 895-905.

17 Williams C. Expert evidence in case of child abuse. Arch Dis Child 1993; 68: 712-4.

\section{Author's response}

I am grateful to Dr Helen Carty for her wise comments on my article, and I am glad of the opportunity to reply to them.

First I wish to point out that since I was invited to write an editorial and not a review, the length of my contribution, including the references, was strictly limited. However, I did find space to refer to Byers comprehensive reviews, ${ }^{12}$ which deal with collagen as well as osteogenesis imperfecta and together contain 592 references, and for those with less mental stamina, a recent discussion on the phenotype and genotype in osteogenesis imperfecta. ${ }^{3}$

I agree with Dr Carty that we await a definitive article in the general paediatric literature - presumably this has not been requested - and I also agree that within paediatrics the general knowledge about current concepts in osteogenesis imperfecta is very varied. One reason may be that in the last two decades most advance in osteogenesis imperfecta has not been made by paediatricians, and is therefore not fully appreciated by them. Dr Carty wants a definitive article, broadly referenced from several sources. In addition to references 1 to 3 , I would suggest to her references 4 to 9 .

Dr Carty's question about the frequency of the gene for abnormal collagen in osteogenesis imperfecta exposes a misinterpretation of the biochemical data. The multicentre linkage study of Dr Sykes and his colleagues showed that in all families studied, the disease was linked to a mutation either in the gene for the alpha 1 chain of type I collagen, that is, COLIA 1 or in the gene for the alpha 2 chain COLIA $2,{ }^{10}$ and this has been widely confirmed, except in an unusual black South African population. ${ }^{11}$ However, the constant association of type I collagen gene mutations with osteogenesis imperfecta established using restriction fragment length polymorphisms does not mean that these specific mutations can always be identified. With increasing knowledge and technique, it is possible to show that the collagen produced by fibroblasts in osteogenesis imperfecta is abnormal either in quality or quantity or both, in up to $85 \%$ of cases. This does not imply that the remaining $15 \%$ do not have a collagen mutation; it merely means that we cannot detect these mutations by available methods. One aim of current research is to develop methods to identify such mutations rapidly and correctly without the need to sequence the whole collagen gene, a prohibitively expensive and tedious procedure.

I am sorry that I have not referenced the statement that normal people have normal collagen. I erroneously considered this to be self evident. When we and others began to look at dermal collagen in osteogenesis imperfecta more than two decades ago, ${ }^{12-14}$ we did of course look at normal (control) subjects with no evidence of connective tissue disorders, and it was against this background that the changes in osteogenesis imperfecta were first identified. Since then, many hundreds of fibroblast cultures from phenotypically normal people have been established, and their collagen synthesis analysed. Such fibroblast lines are always used as controls for the study of collagen mutations and the type and quality of collagen produced by them is well established. I do not know of any accounts of clearly abnormal type I collagen in phenotypically normal persons.

Dr Carty would like to know more about the genetics of osteogenesis imperfecta. Unravelling this has not been easy; luckily the main points are reviewed by Sykes who 